\title{
Essai de reforestation de la Pampa vénézuélienne
}

\author{
par R. FIASSON
}

Tous les ans, au mois de mai, au Venezuela, une fête de l'arbre à laquelle participent les pouvoirs publics et les enfants des écoles coûte à la Nature environ 200.000 arbres.

Dans cette seule journée, il est détruit, dans ce pays, un plus grand nombre d'arbres qu'il n'en est planté dans toute l'année.

En dehors de cette cause de diminution de la forêt, la culture d'une part, I'élevage des chèvres dans certaines régions d'autre part, et enfin les incendies périodiques des savanes durant toute la saison sèche, ont transformé peu à peu la plus grande partie de cette région en une contrée désertique.

Au mois de mai, le jour de la fête de l'arbre, les enfants, sous la conduite de leurs maitres, vont en groupe arracher de jeunes plants vigoureux et les transportent autour des écoles et sur les places publiques. Cette opération est effectuée avec une absence totale de technique et de soins. Une semaine après, il ne reste plus que des branches desséchées qui s'inclinent sous la poussée du vent brûlant du Nord-Est. Si l'on tire sur un de ces vestiges, on s'aperçoit que l'arbuste avait été sorti brutalement sans mêrne prendre l'élémentaire précaution de respecter ses racines.

Inutile d'ajouter qu'en dehors de cette brutale sollicitude d'un jour dont la nature se passerait volontiers, ni l'enfant ni le maître n'accordent plus jamais la moindre attention à leurs sujets transplantés. Au cours de quatre années passées au Venezuela, nous avons en vain cherché un seul résultat heureux de la fête de l'arbre. Les discours sont loin des réalités.

La fête de l'arbre se solde en définitive par une destruction organisée qui s'ajoute à la destruction journalière désorganisée qui, elle, au moins, a l'excuse de l'ignorance ou de la nécessité.

Il est bien évident que les procédés primitifs de la grande majorité des agriculteurs vénézuéliens ne peuvent aboutir qu'à la disparition progressive de la forêt, l'unique engrais étant représenté par les cendres des arbres. Le paysan vénézuélien qui accroche son champ de mais sur les pentes les plus escarpées des Andes augmentant un peu plus l'érosion de la montagne, ou qui l'enclave dans un bosquet des «llanos », diminuant ainsi la maigre végétation de la pampa, n'a pas le choix. Si l'on veut l'ooliger à cultiver ailleurs et avec des méthodes moins primitives, il faudrait d'abord lui fournir des tracteurs, des engrais et l'irrigation dont cette terre a besoin.

Le probleme est souvent un problème d'ignorance, mais pas toujours. Il existe aussi un probleme de moyens.

Dans le but d'enseigner par l'exemple, le paysan des plaines (des llanos) réservées à l'élevage, le Gouvernement vénézuélien décidait, en 1947, la fondation de l'Institut Expérimental et de Recherches dis Llano.

Cet établissement avait donc pour but d'abord de procéder à différents essais de plantations de fourrages, d'arbres et de cultures vivrieres permettant une alimentation plus rationnelle de l'homme et des animaux.

Des laboratoires de bactériologie, de parasitologie et de chimie biologique devaient permettre le recensement des diverses maladies qui entravent lamélioration de l'élevage.

Enfin, I'organisation d'une École Pratique fonctionnant avec une Coopérative pouvait améliorer rapidement les connaissances des éleveurs.

Géographie. - La région choisie pour cette création fut une zone de savane, au Sud de Galabozo (État de Guarico) à $35 \mathrm{~km}$. de cette ville sur la piste de saison sèche qui rejoint San-Fernando de Apure, environ à $9^{\circ}$ latitude Nord et $68^{\circ}$ longitude Ouest de Greenwich.

Cet emplacement présentait le grave inconvénient d'être coupé du reste du pays pendant plusieurs mois de l'année, en hivernage, la route s'arrêtant au rio Guarico, à côté de Calabozo. Ces inconvénients furent atténués, en partie, par un avion que le Ministère mit à notre disposition.

Mais cette région offrait l'avantage d'une communauté agraire comprenant une centaine de familles formant l'agglomération des Bancos, de San-Pedro, et qui constituèrent le premier noyau de la Coopérative. 
Les « llanos » qui sont une caractéristique essentielle du Venezuela s'étendent depuis les montagnes du Sud de Caracas jusqu'aux forêts de la Guyane, depuis les neiges éternelles des Andes jusqu'au delta de l'Orénoque. Ils forment le tiers du pays. Ils constituent les zones d'inondation de l'Orénoque et de ses affluents de la rive gauche. Selon leur altitude, on les classe en "llanos altos» au pied des montagnes et en "llanos bajos » qui forment d'immenses marécages en saison des pluies. On y distingue encore des « mesas» qui sont des parties arides surélevées sur le reste de la plaine et où, en général, il n'y a pas d'eau.

Les llanos semblent s'être formés par apport d'alluvions soulevant peu à peu les terres au-dessus du niveau des mers. Bien qu'à ce sujet les opinions soient partagées, nous avons tout lieu de croire qu'il n'y a pas si longtemps cés pampas étaient amplement boisées, tout au moins dans les llanos du sud et de l'Occident.

Les savanes du Guarico vers la zone des Bancos de San-Pedro sont du type dit "sabanas de mata » parce que la monotonie de la plaine est rompue par de petits bosquets, des groupes de végétation arboricole. On n'y trouve pas cependant le palmier «moriche » : Mauritia flexuosa qui, dans des régions voisines, constitue la végétation type de la pampa. Au pied de ces palmiers sont toujours des marécages d'où sortent des sources d'eau claire, précieuses pour l'abreuvement des troupeaux. On n'y trouve pas non plus le « chaparral 》 Byrsonima crassifolia, arbre des «mesas " qui est le signe de l'absence d'eau et d'un sol particulièrement aride.

Les llanos des Bancos sont donc caractérisés par une juste moyenne d'humidité. Il n'y a pas de cours d'eau, mais la végétation indique une nappe phréatique assez rapprochée de la surface du sol. En effet, on trouve de l'eau à 6 ou 7 mètres. Une pompe à main y puise suffisamment. Pour l'installation d'une éolienne ou d'une pompe centrifuge à moteur, il faut creuser au-dessous de 20 metres.

Climatologie. - Elle présente deux saisons très nettement tranchées. Une saison des pluies qui commence en mai (plus ou moins tardivement selon les années) et qui se termine vers octobre. Il tombe environ $1 \mathrm{~m} .50$ d'eau.

De novembre à avril par contre, la saison sèche est souvent d'une rigueur exceptionnelle, et absolument sans phies. In vent desséchant souffle en permanence de 6 heures du matin à 6 heures du soir. Il vient du Nord-Est. Cette saison est appelée " verano " (été) par opposition à « el invierno" (l'hiver) qui constitue la saison des pluies.

En général, 'il y a interruption dans les pluies pendant quelques jours du mois d'août. C'est le « verano de Agosto » (l'été d'août). Les chutes d'eau les plus violentes ont lieu en juillet. Après l'été d'août, elles' sont moins désordonnées, plus régulières. C'est l'époque la plus favorable aux plantations. Pendant l'hivernage ces llanos s'inondent en partie, soit à cause des rios qui dégorgent leur trop plein, soit à cause de l'eau de pluie qui s'accumule dans les bas-fonds appelés « esteros " par opposition aux parties surélevées et qu'on appelle " bancos ». Les deux tiers de la région des Bancos de San-Pedro sont formés d'esteros. Ils sont dono sous l'eau durant deux ou trois mois, tous les ans.

Ces savanes, en saison sèche, sont régulièrement dévastées par des incendies allumés par les éleveurs dans le but de détruire des reptiles dont le Crotale Crotalus terrificus.qui sont assez nombreux, et des acariens, particulièrement des Ixodes des espèces Amblyomma cayennense et Boophilus 'microplus qui pullulent. Le but de ces incendies est ausi, comme ailleurs dans les régions tropicales, de provoquer la pousse d'un regain plus alibile, de purger leurs animaux avec les cendres; enfin, de faciliter les déplacements.

Ces plaines sont en partie soumises à l'érosion provoquée par la surcharge des animaux autour des points d'eau et à cause du vent violent du Nord-Est. Au sujet de l'érosion, il est utile d'appeler l'attention des pouvoirs publics sur la nécessité d'une refo'restation et d'une protection par des haies d'arbres dans les savanes de Portuguesa, où une politique du riz a transformé en champs de poussière d'immenses pâturages. Les tempêtes de sable de l'Ouest des États-Unis devraient inciter les hommes à la prudence.

En réalité, si durant la saison des pluies, les llanos vénézuéliens constituent un agréable océan d'herbes demi-noyées, en été, sous l'action du vent et des incendies, c'est le royaume désolé de la cendre et de la poussièro.

Nature du sol. - Les expériences de reforestation du llano effectuées dans notre Institut portèrent sur un sol dont la composition est la suivante :

Au-dessus de 30 centimètres, la proportion de sable varie de 36 à $46 \%$, l'argile de 14,4 à $24,4 \%$, le limon de 39,6 à $49,6 \%$.

Entre 30 et 60 centimètres, la proportion de sable devient de 19 à $42 \%$, celle de l'argile de 24,4 à $35,4 \%$, et celle du limon de 30,6 à $45,6 \%$.

Le pourcentage des matières organiques varie de 1,14 à $2,15 \%$. Le $\mathrm{pH}$ se tient entre 5.7 et 6.2 . Ces terrains contiennent :

Azote total : $\%$. . . . . de 0,9 à $3(0,9 / 1,1 / 3)$ Chaux : $\%$.......... de 7,5 à $26(7,5 / 13 / 11 / 26)$

Acide phosphorique assi-

milable : $\%$ o $\ldots \ldots \ldots$ de 4,1 à $10(8,5 / 10 / 4,1 / 10)$

Potasse assinilable .... \% \% $=0$

(Analyse effectuée par le Service des Sols de Maracay). 


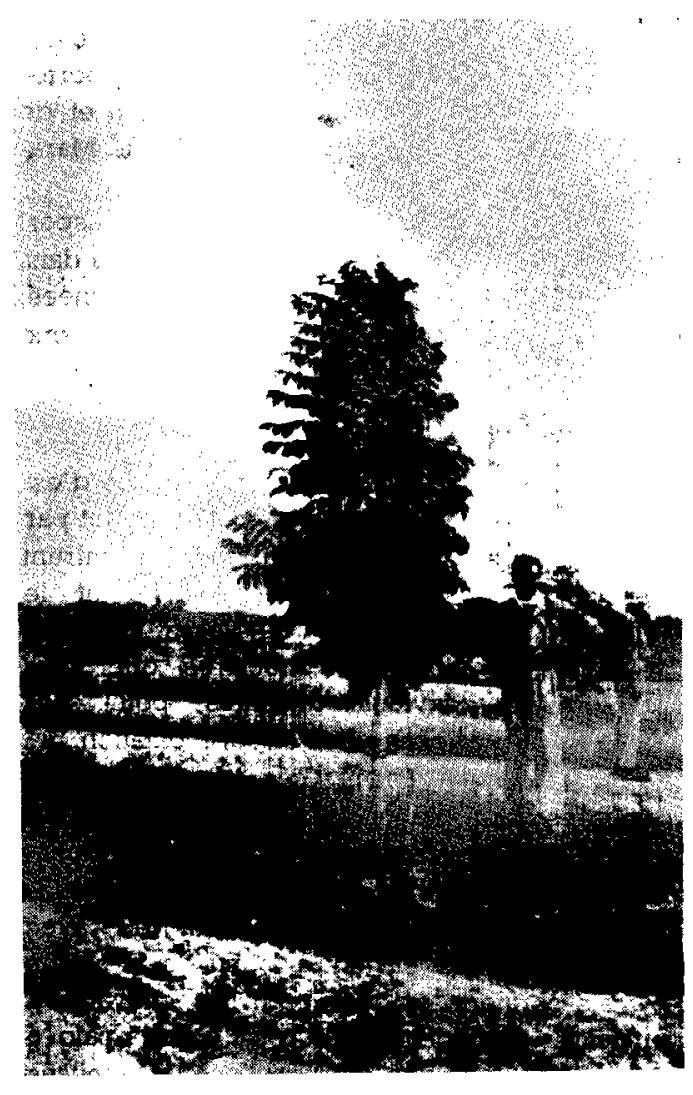

Spathodea campanulata, 15 mois.

Les arbres qui poussent naturellement dans les bosquets épars sur ces savanes sont : l' " aceite " Copaifera officinalis, le "pilon" Andina inermis, le " bucare " (plusieurs espèces du genre Erythrina de la famille des Papilionacées), le "mercure» Couepia guianensis, le «merei»

Anacardium occidentale, le "parapara " Sapindus saponaria, le « saman " Samanea saman, le "manirote» Annona purpurea, le " mata-palo » Ficus sp., le "mata-raton » Gliricidia sepium, le "guayabo» (plusieurs espèces de la famille des Myrtacées), des manguiers, Mangifera indica qui, bien que d'origine étrangère, s'est si completement naturalise qu'on le trouve un peu partout dans le llano; il en existe de nombreuses variétés; le «lechero» (plusieurs espèces d'Euphorbiacées), un palmier « plama llanera " Copernicia tectorum: parfois quelques rares « ara-

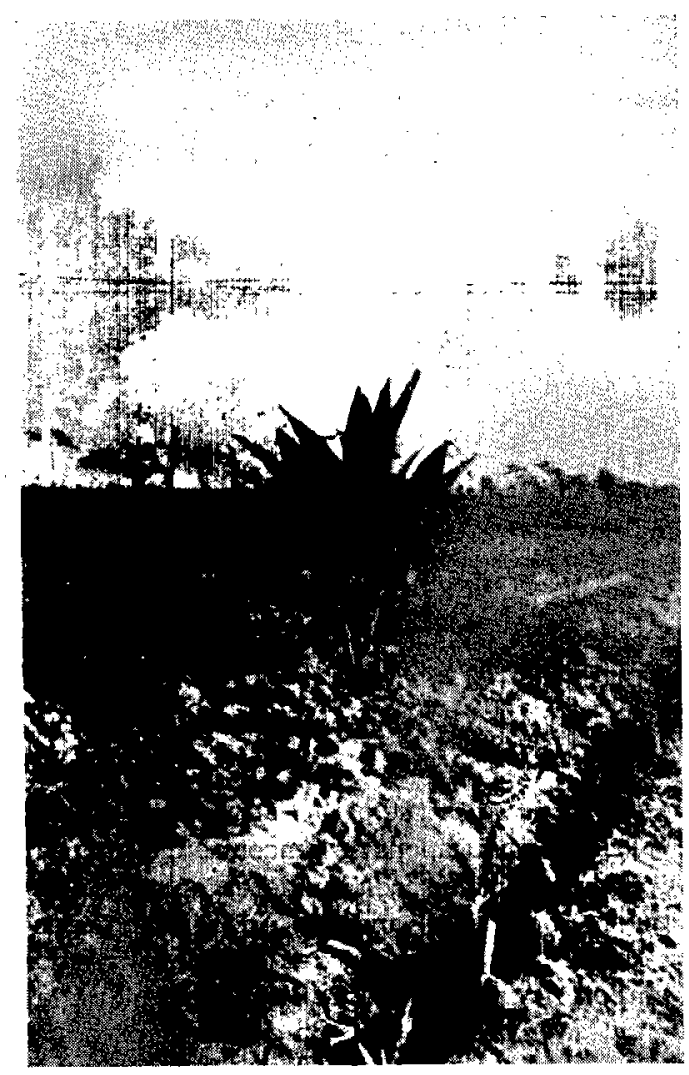

Cocotier, 1 an.

guaney », I'arbre national du Venezuela', plus fréquent sur le "llano alto ", inagnifique touffe blonde de fleurs sans feuilles à la fin de la saison sèche; le "guacimo " (Guazuma ulmifolia), le, " dividive " Caesalpinia Coriceria, le "jobo» Spondias

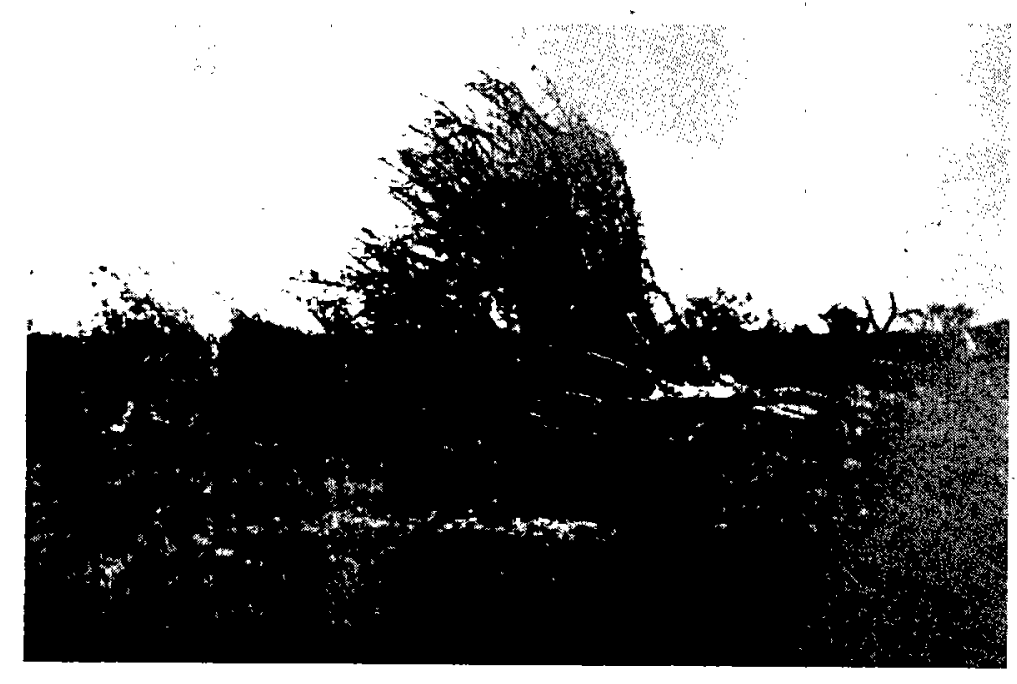

Fin de Mexpérimentation (destruction par le Gouvernement militaire). 


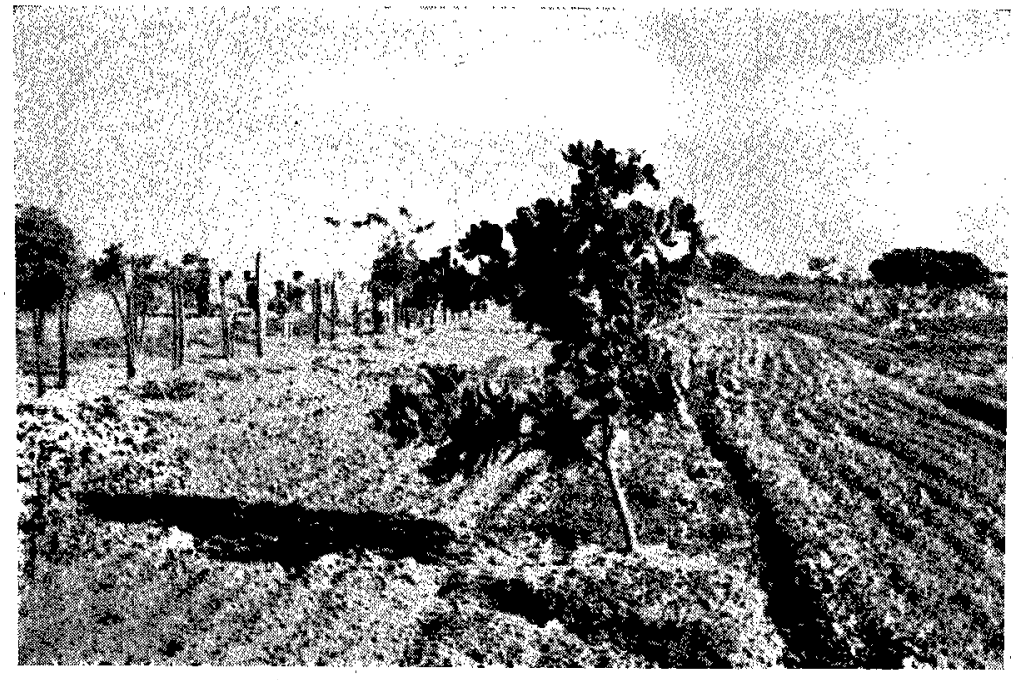

Anacardium occidentale ( $1 \mathrm{~m} .50,1$ an après semis).

Les essais furent effectués avec de jeunes plants rapportés des stations de Caracas et de Rancho-Grande près de Maracay.

Pour éviter le transport difficile de jeunes arbres dans des régions aussi éloignées, nous commençâmes, en même. temps, à créer nos propres pépinières.

Enfin, sur place, nous trouvâmes un certain nombre d'espèces qui se reproduisent par boutures et qui nous permirent d'édifier des haies tout: le long des barrières des 100 premiers hectares que nous avions délimités pour nos champs d'expérimentation.

Parmi ces dernières, les

lutea, le " ciruelo » Spondias purpurea...

Près des habitations on rencontre d'autres espèces soit omementales, soit fruitières en plus des manguiers, goyaviers, anonnes, jobos et ciruelos déjà signalés, en particulier des cocotiers qui donnent un bon rendement malgré l'éloignement de la mer; mais les indigènes ont soin de les arroser avec de l'eau salée ou du petit lait. On y trouve en particulier une grande variété d'anonnes, comme le " guanabana » Anonna muricatus, le "rinon" A. cinerea, le "chirimoya » $A$, cherimolia, " anon » A. squamma. Il y a toujours quelques arbres de « onoto » Bixa orellana qui colore les bouillons et les sauces en ocre, Ie «tapara 》Crescentia cujete dont l'écorce du fruit sert ì fabriquer les calebasses, le "nispero » Achras sapota, l'une des meilleures nèfles; «arbol de pan » ou «pan de pobre » Artocarpus communis, parfois quelques avocatiors, Persea americana avec de nombreuses variétés et, beaucoup plus répandu, le papayer Carica papaya. Le "manei " Mammea americana foumil un fruit extrêmement parfumé, malheureusement il est rare et peu connu, ainsi que la barbadine ou " parcha » de la famille des Passiflores. Les orangers et citronniers sont communs. Sauf ces demiers, ainsi que les manguiers déjà signalés, toutes ces espèces sont indigènes.

Bien entendu, dans les galeries forestières' sur les rives des cours d'eau, la flore est beaucoup plus variée.

Expérimentation. - Nos expériences eurent lieu pendant l'hivernage de l'annee 1948 qui fut particulièrement violent et auquel succéda une saison sèche d'une extrême rigueur. plus utilisées sónt le « mata-raton 》 Gliricidia sepium, une papilionacée qui "pousse rapidement et qui, convenablement taillée, forme des haies assez épaisses. Les feuilles sont mangées avec avidité par les bovins surtout à la fin de la saison seche, mais seraient toxiques pour les chevaux.

"Ciruelo » Spondias purpurea, « mata-palo » et Ficus dendrocida, "lechero » (Nombreuses espèces de la famille des Euphorbiacées, entrent aussi dans cette catégorie et sont plus ou moins utilisées avec quelques autrés comme les « cardones " (Cactacées terrestres en forme de colonnes) et l'arbre à calebasses.

Sans aucun doute lés meilleurs résultats, dans les llanos, sont obtenus avec la première espèce Gliricidia sepium, mais il faut avoir soin de protéger les premières pousses contre la voracité des bovidés.

Parmi les arbres fruitiers qui furent plantés dans les terrains de l'Institut, signalons 250 orangers, citronniers, mandariniers et grape-fruit, greffés, de deux ans et mis en terre au début des pluies. Ils souffrirent beaucoup de l'excès d'humidité et 80 d'entre eux se desséchèrent. Ils furent remplacés à la fin de l'hivernage. Il faut eviter d'effectuer des plantations avant l'été d'août.

2.000 orangers appelés improprement de Valence, arbustes de un an et non greffés furent plantés entre le 15 et la fin d'août, dans un terrain légèrement plus surélevé. Nous n'en perdîmes pas plus de 100 .

Tous ces arbres furent arrosés pendant la saison sèche.

A la fin du mois d'août, nous effectuâmes la plantation de 100 noix de coco, 10 avec sel et 90 sans sel. 100 autres furent placées dans l'eau, l'écorce coupée du côté du germe selon la coutume des propriétaires 
des cocoteraies de la côte de la mer des Caraíbes.

Ces dernières germèrent plus tôt, mais les résultats au point de vue résistance et rapidité de croissance de l'arbre, furent meilleurs avec la première méthode. Le sel ne parut pas avoir d'action.

Pour la reforestation de la pampa tropicale, les essais furent faits avec les plants suivants :

"Urape » Bauhinia multinervia : arbre qui peut atteindre 10 à 12 mètres, épineux.

Ils se révélèrent très résistants à l'excès d'humidité comne à l'extrême sécheresse. Malheureusement ils furent presque tous détruits par les

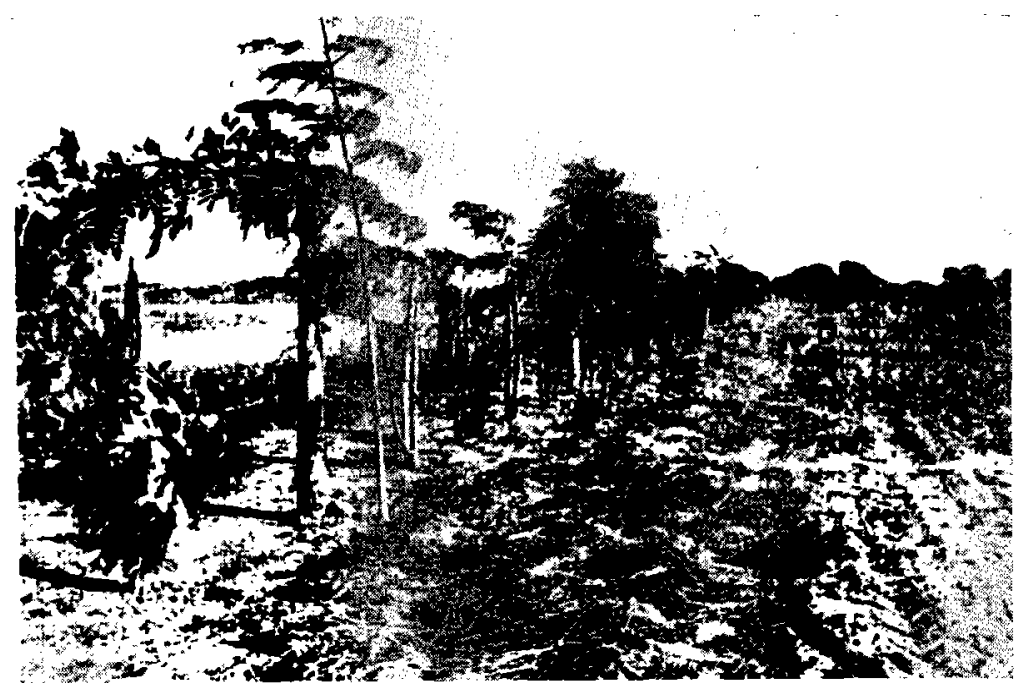

Haie de Gliricidia sepium

(au premier plan à gauche, Delenir regia, $2 \mathrm{~m}$. 50, 15 mojs après semis). «bachacas » (fourmis-parasols)

qui ont une véritable prédilection pour les petites feuilles fragiles et tendres de l'urape.

La résistance de cette espèce est telle que les arbustes reprirent leurs feuilles à plusieurs reprises après avoir été complètement tondus par les fourmis. Cependant, après plusieurs attaques ils se desséchèrent.

Il nous fut aisé de détruire les grandes colonies des insectes prédateurs avec du sulfure de carbone. Mais il est impossible de s'attaquer avec succès à toutes les jeunes familles, très actives, et qui n'ont pas besoin de plus d'une nuit pour réaliser l'épluchage de l'arbre.

«Araguaney » Tecoma chrysantha, l'arbre national du Venezuela, qui pousse dans des régions voisines, se révéla, au contraire, un arbre extrêmement fragile. L'humidité d'une part et la sécheresse ensuite, amenèrent rapidement la disparition de la plantation. Les quelques-uns qui résistèrent ne se développèrent pas.

«Casoarina » Casuarina quadrivalvis et C. equisetifolia, introduits dans un grand nombre de régions tropicales comme plants d'ornementation et pour la reforestation des terrains incultes; ces espèces, d'origine australienne, bien que les conditions fussent très éloignées de leur propre milieu, se montrèrent très résistantes et d'une grande possibilité de développement.

Plantés au début d'août, ces jeunes arbustes, qui ne dépassaient pas 15 centimetres, 10 mois plus tard atteignaient $1 \mathrm{~m} .75$ avec un tronc très droit et très fort. Cependant cette rapidité de croissance oblige à les soutenir car le vont du Nord-Est les briscrait. Mais le plus grave danger pour l'extension de cette espece est constitué par les léporidés. Le petit lievre sud-américain trouve un plaisir particulier à en sectionner le tronc lorsque l'arbre esi encore iragile. Il est necessaire de les protéger avec du grillage ce qui limite singulièrement leur utilisation.

"Pomarosa » Jambosa vulgaris. De la famille des Mirtacées. Originaire de l'Inde. Très utilisé comme arore d'ornemont pour son ombre et comme brisevent. Il est maintenant demi-naturalisé au Venezuela. Scn fruit d'une belle couleur rouge est comestible et possède un vague parfurn de rose.

Ces arbustes se developpèrent très rapidement cans ure terre argileuse et regorgeant d'eau. Mais, a ¿a saison seche, ils ne continuerent pas leur progression comme les pins australiens. Néanmoins, iis résisterent mieux que la plupart des espèces incigienes.

«Saman 》Samanea saman déjà signalé comme un ariore des parties humides de la région.

C'est un arbre magnifique, l'un des plus beaux des terres chaudes du Venezuela. De la famille des Mimosacées, de tronc court en relation avec une couronne énorme très étendue. Utilisé autrefois comme ombrage pour les plantations de café, mais il épuise rapidement le sol et au temps de la floraison, I'abonaance des fleurs est telle qu'elle écrase les arbustes de café et de cacao sous leur poids. Bien qu'il soit indigène, il est extrêmement fragile. Au début des pluies on rencontre des milliers d'arbustes nés des graines tombées, mais dès que les pluies s'accentuent, ils disparaissent et les samans'adultes qui se sont développés sans l'aide de l'homme sont rares. Il est très utile pour l'alimentation du bétail en fin de saison sèche car les animaux des espèces 
bovine, ovine, caprine et porcine sont très friands de ces gousses.

Mais lorsqu'il est protégé de l'excès d'humidite et arrosé en saison sèche comme nous pûmes le faire pour quelques centaines de plants que nous trouvâmes près de parcs à cochons et que nous plaçâmes tout le long d'une allée, ils sont capables. d'un développement inoui. Certains d'entre eux dépassaient 2 mètres au bout de 14 mois.

" Eucalyptus », Eucalyptus sp. 4 ou 5 espèces australiennes ont été introduites au Venezuela par les cons'tructeurs des chemins de fer. C'est l'espèce E. Globulis qui est la plus répandue.

Les llanos constituent une dure épreuve pour ces arbres qui se sont acclimatés un peu partout sous les tropiques. Les terrains argileux, véritables bourbiers en saison des pluies ne leur conviennent pas. Ils supportèrent assez mal aussi la longueur exceptionnelle de la saison sèche.

« Tulipan africano »" ou Caoba de Santo. Domingo », Spathodea campanulata. De la famille des Bignoniacées. Arbre ormemental, originaire du Gabon, qui, malgré le nom de « caoba » sous lequel il est parfois, connu n'a rien de commun avec l'acajou. Ces arbres, convenablement soignés, se développent avec une rapidité extraordinaire. Si l'on peut l'arroser durant la saison sèche, c'est l'espèce qui donne le plus de satisfaction par la rectitude et la robustesse de son tronc, son feuillage épais et son incroyable rapidité de croissance. Certains exemplaires dans les terrains de l'Institut dépassèrent 3 mètres en 14 mois.

Nous en arrivons maintenant aux deux espèces qui nous ont paru les plus aptes pour une politique générale de reforestation.

De ces deux espèces, l'une est autochtone, c'est le «merei 》 (Anacardium occidentale), l'autre a été introduite, c'est le flamboyant (Delonix regia), originaire de Madagascar.

Ces deux espèces ont l'avantage de pouvoir se développer rapidement par semis, de présenter une résistance très grande à l'excès de l'humidité et à la sécheresse prolongée, de n'être pas attaquées par les animaux domestiques, de ne pas attirer les rongeurs qui sectionnent les jeunes plants, enfin, d'être des arbres décoratifs.

L'anacardier, appelé aussi « pauji » sur l'Orénoque ou « maranon » vers Maracailbo, est un arbre de la famille des Anacardiacées. C'est un arbre relativement petit (cependant, nous avons vu des spécimens de plus de 10 mètres de hauteur et d'un diamètre de 40 à 50 centimètres), à feuilles alternées, ovales, obtuses, à fleurs polygames, pentamères, à ovaire unicellulaire et uniovulé (Pittier).

Il réussit dans des sols qui ne conviennent à aucune autre culture. Se propage par semis. Avant le semis, on fait sécher les graines au soleil pendant une dizaine de jours.

A 2 ans, la première floraison et la première fructification sont possibles. Mais souvent il faut attendre la troisième année.

L'anacardier présente sur le flamboyant les avantages suivants : ses feuilles ne sont pas attaquées par les fourmis-parasols alors que celles du flamboyant, plus fragiles et plus tendres, sont parfois découpéés et emportécs (ellcs sont toutcfois moins appréciées par ces insectes que le feuillage de l'urape); il donne aussi un feuillage beaucoup plus fourni; enfin, les indigènes de la Pampa apprécient beaucoup ses fruits et sa graine en dehors de quelques autres propriétés de cet arbre.

L'écorce et les feuilles sont riches en tanin et peuvent être utilisées pour le tannage local. Avec ces dernières, on prépare aussi des lotions astringentes utilisées contre les maux de gorge. De l'écorce suinte une gomme soluble qui peut constituer un substitut de la gomme arabique.

Le bois, de couleur rougeâtre, d'un poids spécifique de 0,50: est assez résistant. On l'utilise en charpenterie et surtout en ébénisterie. La cendre qu'on en retire contient un pourcentage élevé de potasse.

De la partie juteuse du fruit, c'est-à-dire du pédoncule, on peut extraire un vin qui est considéré par les « llaneros » comme un des meilleurs antidysentériques connus.

Ce pédoncule charnu qui supporte le fruit sec indéhiscent, réniforme, est très juteux, de couleur rouge ou jaune. Sa saveur est âpre et il est difficile de le manger frais, mais on peut en faire des gelées agréables.

La graine qui surmonte 'ce pédoncule possède une enveloppe extérieure qui' contient un liquide huileux, très âcre, de couleur marron, qui noircit à l'air et qu'on peut utiliser comme encre indélébile. Ce liquide est encore employé comme vésicant et médicamont contro la lòpre; pour dótruire los verrues et les dents cariées (sic).

Il n'est donc pas possible de consommer ces graines fraiches, mais grillées, elles sont de saveur agréable. Elles remplacent les amandes et on en exporte des quantités considérables aux États-Unis et en Europe. Ce n'est d'ailleurs ni du Venezuela ni de l'Amérique tropicale qu'elles proviennent, mais de certaines régions des Indes Orientales où le « merei » a été introduit et cultivé en grandes plantations.

On en trouve quelques arbres à Madagascar, qui ont dû être introduits de ces mêmes régions.

Aux Etats-Unis, on fait une grosse consommation de ces amandés grillees et on en exporte dans le monde entier mélangées avec des amandes, des 
noix du Brésil, des noisettes, des arachides, des noix, etc., et présentées dans des boîtes de fer.

Les graines mises en terre au début de la saison des pluies se développent rapidement et les plantes atteignent 50 centimètres à la fin de l'hivernage. Cette taille et sans doute le développement des racines leur permettent de supporter la première saison sèche sans besoin d'arrosage. Le pourcentage des pertes est relativement faible durant les premiers mois de secheresse. Il ne dépasse guère $10 \%$. Après la deuxième saison des pluies, l'arbre est pratiquement sauvé.

Nous placions 3 graines dans chaque trou à 15 centimètres de profondeur. Il est facile, au bout d'un an, d'éliminer les plants les moins vigoureux si plusieurs ont germé.

Une plantation sur une grande échelle fut faite à l'Institut du llano au debut de l'hivernage 1949 (mai).

Sur un terrain labouré, hersé, bien préparé à l'avance, d'une étendue d'un peu plus de 12 hectares, nous plantâmes 5.000 anacardiers. Entre les rangées espacées de 5 mètres, des cultures de haricots et de Crotalaria furent effectuées.

Le pourcentage d'arbustes s'étant bien développé au bout de 3 mois, atteignit $75 \%$. Malheureusement, cette plantation fut détruite au tracteur (avec, d'ailleurs, toutes les pépinières, la plus grande partie des autres essais et les grands arbres existant avant notre arrivée) par la nouvelle direction établie par le Gouvernement militaire.

Conclusion. - Effectuées à l'Institut du llano, dans les savanes du Guarico, au Venezuela, et malgré qu'elles aient été interrompues alors que les essais ne faisaient que commencer, on peut cependant tirer la conclusion que le merei « Anacar- dium occidentale ", à cause de sa rapidité de croissance, la simplicité de sa plantation par graines, sa résistance aux facteurs destructifs de ces régions (qui sont constitués habituellement par l'excès d'humidité en hivernage, l'extrême sécheresse en d'autres mois, ainsi que par les fourmis-parasols), l'intérêt que les habitants des llanos portent à cet arbre pour ses fruits, son bois et autres utilisations industrielles ou pharmaceutiques qu'on peut tirer du fruit, de l'écorce ou des feuilles, cet arbre est d'un précieux secours pour une politique rationnelle et suivie de reforestation du llano.

Il pourrait, peut-être aussi, présenter un intérêt pour la reforestation dans d'autres parties du globe, les terrains dans lesquels il se développe normalement n'étant pas des terrains particulièrement riches, bien au contraire.

Selon la Chambre d'Agriculture du Tonkin (Revue Trop. Agric. 1937), l'anacardier existe à l'état subspontané à Ceylan, mais on en trouve aussi cultivé dans quelques plantations dans la zone seche comme dans la zone humide. Les amandes des fruits étaient vendues par les femmes indigènes sur le marché de Colombo.

En 1937, dans la région de Monnar, on comptait 200 hectares de petites plantations ne dépassant pas chacune I hectare. La production, annuelle des noix s'élevait à 110 tonnes.

Les plantations sont faites par semis. Les graines séchées sont semées, soit dans des paniers (Kuddans) pour faciliter le transport des seedlings, soit en pépinières. Puis on place les jeunes plantes par 2 ou 3 dans le même trou, à $12 \times 12$ mètres en tous sens.

D'après cette revue, le rendement varie de 4.000 à 6.000 noix par arbre et par an. 